Pacific Journal of Mathematics

RELATIVE NULLITY FOLIATIONS AND INDEFINITE 


\title{
RELATIVE NULLITY FOLIATIONS AND INDEFINITE ISOMETRIC IMMERSIONS
}

\author{
Kinetsu Abe AND Martin Magid
}

\begin{abstract}
This paper investigates the relative nullity distribution of an indefinite Riemannian manifold isometrically immersed into an indefinite space form.
\end{abstract}

Introduction. In this paper we investigate the relative nullity distribution of an indefinite Riemannian manifold isometrically immersed into an indefinite space form. This distribution is totally geodesic and gives rise to a Ricatti-type differential equation along a geodesic in a leaf of the distribution.

This differential equation is applied in several ways to estimate the index of relative nullity $\nu$ for geodesically complete, connected, Lorentzian submanifolds $M_{1}^{n}$ of $\tilde{M}_{1}^{n+1}(c)$, the Lorentzian sphere. These applications extend the work of Abe [1], [2], [3], Ferus [7], [8], and others to the setting of indefinite manifolds. Some of the work in $\$ 2$ was obtained previously by Graves [10] in the codimension one case and by M. Dajczer. In particular Theorem 2 was conjectured by Dajczer [5].

Sections 1 and 2 lay the groundwork and derive the Ricatti-type differential equation. In $\S 3$ an integer $\nu_{n}$ is defined and it is shown that if $M_{1}^{n}$ is as above and if $\nu>\nu_{n}$ then $M_{1}^{n}$ is totally geodesic. This integer is used to formulate a geometric condition which guarantees that a complete connected hypersurface of $S_{1}^{n}(c)$ is totally geodesic. We also estimate $\nu$ given a natural condition on the space-like Ricci curvature of the submanifold. In [6] other conditions on Ricci curvature are given.

The general scheme of our investigation is very similar to that of the Riemannian case as formulated in the papers mentioned above. However, there are a few basic and non-trivial differences from the Riemannian case. These differences are due to the indefinite metric and are to be overcome. Therefore, we think it worthwhile to include the details of the proofs for most of our results.

1. Preliminaries. An indefinite Riemannian manifold $M_{s}^{n}$ of dimension $n$ is a connected manifold with a non-degenerate metric in each 
tangent space. The metric can be written

$$
d s^{2}=-\left(d x^{1}\right)^{2}-\cdots-\left(d x^{s}\right)^{2}+\left(d x^{s+1}\right)^{2}+\cdots+\left(d x^{n}\right)^{2}
$$

at every point of $M^{n}$. In this case, we say the signature of $M^{n}$ is $(s, n-s)$ and write $M_{s}^{n}$.

If $f: M_{s}^{n} \rightarrow \tilde{M}_{t}^{n+k}$ is an immersion and the metric induced on $M^{n}$ is non-degenerate, $f$ is an isometric immersion of $M_{s}^{n}$ with this metric into $\tilde{M}_{t}^{n+k}$. Denote by $D$ the torsion-free metric connection on $\tilde{M}_{t}^{n+k}$. $D$ induces a torsion-free, metric connection $\nabla$ on $M$ as follows:

$$
D_{X} Y=f_{*}\left(\nabla_{X} Y\right)+\alpha(X, Y),
$$

where $X$ and $Y$ are tangent vectors on $M, f_{*}\left(\nabla_{X} Y\right)$ is the tangential component and $\alpha(X, Y)$ is the normal component. $\alpha$ is called the second fundamental form of $f$.

Given a field of unit normal vectors $\xi$ on $f\left(M_{s}^{n}\right)$ we can define a field of endomorphisms $A_{\xi}$ on $M$ by

$$
D_{X} \xi=-f_{*}\left(A_{\xi} X\right)+\nabla_{X}^{1} \xi
$$

where $-f_{*}\left(A_{\xi} X\right)$ is the tangential component. $A_{\xi}$ is called the shape operator associated to $\xi$.

We denote by $N(x)$ the set of all normal vectors to $f\left(M_{s}^{n}\right)$ at $f(x)$. The metric on $\tilde{M}_{t}^{n+k}$ and $M_{s}^{n}$ is denoted by $\langle$,$\rangle . As usual, R($,$) denotes$ the curvature tensor of $M_{s}^{n}$. For the sake of future use, we list the following:

$$
\begin{aligned}
R(X, Y) Z= & \tilde{R}(X, Y) Z+\sum_{p=1}^{k}\left\langle\xi_{p}, \xi_{p}\right\rangle\left[\left(A_{\xi_{p}} X\right) \wedge\left(A_{\xi_{p}} Y\right)\right] Z \\
= & \tilde{R}(X, Y) Z+A_{\alpha(Y, Z)} X-A_{\alpha(X, Z)} Y \\
& \text { (Gauss equation); }
\end{aligned}
$$

(1.4) $S(X, Y)=\sum_{i=1}^{n}\left\langle X_{i}, X_{l}\right\rangle\left\langle R\left(X_{l}, X\right) Y, X_{i}\right\rangle \quad$ (Ricci tensor);

$\tilde{R}($,$) is the curvature tensor of \tilde{M}_{t}^{n+k} ; \xi_{p}$ 's form an orthonormal base for $N(x)$; and $X_{l}$ 's form an orthonormal base for $T_{x} M$, the tangent space of $M_{s}^{n}$ at $x$.

2. Relative nullity. If $f: M_{s}^{n} \rightarrow \tilde{M}_{t}^{n+k}$ is an isometric immersion between indefinite Riemannian manifolds we define the relative nullity space at $x, T^{0}(x)$, to be

$$
T^{0}(x)=\left\{X \in T_{x}(M): A_{\xi} X=0 \quad \forall \xi \in N(x)\right\} .
$$


The orthogonal complement $\left[T^{0}(x)\right]^{\perp}$ of $T^{0}(x)$ in $T_{x} M$ is denoted by $T^{1}(x)$.

Proposition 1. $T^{0}(x)=\left\{X \in T_{x}(M): \alpha(X, Y)=0 \forall Y \in T_{x}(M)\right\}$.

Proof. It is obvious.

Proposition 2. $T^{1}(x)=\operatorname{span}\left\{A_{\xi} Y\right\}$ for $\xi \in N(x), Y \in T_{x}(M)$.

Proof. Given any $\xi \in N(x), Y \in T_{x}(M)$ and $X \in T^{0}(x),\left\langle X, A_{\xi} Y\right\rangle$ $=0$, so $A_{\xi} Y \in T^{1}(x)$.

On the other hand, suppose $Z \in T_{x}(M)$ satisfies $\left\langle Z, A_{\xi} Y\right\rangle=0$ for all $\xi, Y$ as above. Then $\alpha(Y, Z)=0$ for all $Y$ and $Z \in T^{0}(x)$. This means that $\left[\operatorname{span}\left\{A_{\xi} Y\right\}\right]^{\perp} \subset T^{0}(x)$ so that $\left[\operatorname{span}\left\{A_{\xi} Y\right\}\right] \supset T^{1}(x)$.

The dimension $\nu(x)$ of $T^{0}(x)$ is called the relative nullity of the immersion at $x$. The minimum value of $\nu(x)$ on $M$ is called the index of relative nullity and is denoted by $\nu_{0}$.

THEOREM 1. Assume that $\tilde{M}$ is a space form and let $G$ denote the set of points in $M$ where $\nu(x)=\nu_{0}$. Then

(1) $G$ is an open subset of $M$;

(2) $x \rightarrow T^{0}(x), x \in G$ is a differentiable and involutive distribution in $G$;

(3) the foliation $T^{0}$ is totally geodesic in $M$; and

(4) each leaf of $T^{0}$ is immersed as a totally geodesic submanifold of $\tilde{M}$.

Proof. (1) Pick a point $x_{0} \in G$ and a basis $T_{x_{0}}(M),\left\{Y_{1}, \ldots, Y_{\nu_{0}}\right.$, $\left.Y_{\nu_{0}+1}, \ldots, Y_{n}\right\}$, such that, for some $\xi_{j}\left(x_{0}\right),\left\{A_{\xi_{j}} Y_{\nu_{0}+\jmath}\right\}$ forms a basis of $T^{\mathrm{l}}\left(x_{0}\right)$. Extend $Y_{1}, \ldots, Y_{n}$ and $\xi_{1}, \ldots, \xi_{n-\nu_{0}}$ smoothly in a neighborhood of $x_{0}$. The set $\left\{A_{\xi}, Y_{\nu_{0}+j}\right\}$ remains linearly independent in a neighborhood of $x_{0}$. Therefore in a neighborhood of $x_{0}$ the dimension of $T^{0}\left(x_{0}\right)$ must be less than or equal to $\nu_{0}$, and so equals $\nu_{0}$.

(2) It can be shown that $T^{0}$ is a smooth distribution on $G$ by noting that $T^{0}=\bigcap_{j=1}^{k} \operatorname{ker} A_{\xi}$ and that this intersection has constant rank on $G$.

(3) We use Codazzi's equation to see that $T^{0}$ is totally geodesic. Let $Y, Z$ be vector fields in $T^{0}$. For all $X$ in $T M$ and normal vectors $\xi$ we have

$$
\nabla_{X}\left(A_{\xi} Y\right)-A_{\xi}\left(\nabla_{X} Y\right)-A_{\nabla_{\frac{1}{X}} \xi} Y=\nabla_{Y}\left(A_{\xi} X\right)-A_{\xi}\left(\nabla_{Y} X\right)-A_{\nabla_{\frac{1}{Y}} \xi} X .
$$


This reduces to

$$
-A_{\xi}\left(\nabla_{X} Y\right)=\nabla_{Y}\left(A_{\xi} X\right)-A_{\xi}\left(\nabla_{Y} X\right)-A_{\nabla_{Y}^{\frac{1}{}}} X .
$$

Taking the inner product of both sides with $Z$ yields $0=\left\langle\nabla_{Y}\left(A_{\xi} X\right), Z\right\rangle$. $\left\langle A_{\xi} X, Z\right\rangle=0$ can be differentiated in the direction of $Y$.

$$
0=Y\left\langle A_{\xi} X, Z\right\rangle=\left\langle\nabla_{Y}\left(A_{\xi} X\right), Z\right\rangle+\left\langle A_{\xi} X, \nabla_{Y} Z\right\rangle \text {. }
$$

This gives $\left\langle A_{\xi} X, \nabla_{Y} Z\right\rangle=0$, i.e., $\nabla_{Y} Z \in T^{0}$. This shows $T^{0}$ is totally geodesic. Examining Codazzi's equation with $Y$ in $T^{0}$ and $\xi, X$ as above gives

$$
-A_{\xi}\left(\nabla_{X} Y\right)=\nabla_{Y}\left(A_{\xi} X\right)-A_{\xi}\left(\nabla_{Y} X\right)-A_{\nabla_{Y}^{\frac{1}{Y}}} X
$$

so that

$$
\nabla_{Y}\left(A_{\xi} X\right)=A_{\xi}\left(\nabla_{Y} X\right)+A_{\nabla_{Y}^{1} \xi} X-A_{\xi}\left(\nabla_{X} Y\right) .
$$

This shows that $T^{1}$ is also parallel along $T^{0}$.

For (4), notice that

$$
D_{Y} f_{*}(Z)=f_{*}\left(\nabla_{Y} Z\right)+\alpha(Y, Z)=f_{*}\left(\nabla_{Y} Z\right) .
$$

Next we define a complementary distribution $T^{c}(x)$ in a neighborhood of $y(t), t \in[a, b)$ where $y(t)$ is a geodesic in a leaf of $T^{0}$. The distribution is complementary in the sense that $T^{c}(x) \oplus T^{0}(x)=T_{x}(M)$.

If, at a fixed point $y(0), T^{0}(y(0))$ is non-degenerate then $T^{0}(x)$ is non-degenerate for all points $x$ near $y(0)$. Along a geodesic $y(t)$ in a leaf of $T^{0}, T^{0}(y(t))$ remains non-degenerate since $T^{0}(y(t))$ is parallel along the geodesic. Therefore, $T^{0}(x)$ is non-degenerate in a neighborhood of the geodesic. In this case set $T^{c}(x)=T^{1}(x)$.

If $T^{0}(y(0))$ is degenerate we use the following procedure. At $y(0)$ choose a pseudo-orthonormal basis

$$
\left\{L_{1}(0), \ldots, L_{r}(0), E_{1}(0), \ldots, E_{\nu_{0}-r}(0)\right\} \text { of } T^{0}(y(0))
$$

and

$$
\left\{L_{1}(0), \ldots, L_{r}(0), F_{1}(0), \ldots, F_{n-\nu_{0}-r}(0)\right\} \text { of } T^{1}(y(0))
$$

so that

$$
\left\langle L_{i}(0), L_{j}(0)\right\rangle=0=\left\langle L_{i}(0), E_{k}(0)\right\rangle=\left\langle L_{i}(0), F_{l}(0)\right\rangle
$$

and the $E_{k}(0)$ and $F_{l}(0)$ form an orthonormal set. Add $\left\{\hat{L}_{1}(0), \ldots, \hat{L}_{r}(0)\right\}$ so that each $\hat{L}_{i}(0)$ is perpendicular to $E_{k}(0)$ and $F_{l}(0),\left\langle\hat{L}_{i}(0), \hat{L}_{j}(0)\right\rangle=0$ and $\left\langle L_{i}(0), \hat{L}_{j}(0)\right\rangle=-\delta_{i j}$. 
Denote the parallel extension of this basis

$$
\begin{aligned}
& \left\{L_{1}(0), \ldots, L_{r}(0), E_{1}(0), \ldots, E_{\nu_{0}-r}(0),\right. \\
& \left.\quad F_{1}(0), \ldots, F_{n-\nu_{0}-r}(0), \hat{L}_{1}(0), \ldots, \hat{L}_{r}(0)\right\}
\end{aligned}
$$

along $y(t)$ by $\left\{L_{1}(t), \ldots, \hat{L}_{r}(t)\right\}$.

Assume, without loss of generality, that $\vec{y}(0)$ is one of

$$
\left\{L_{1}(0), \ldots, L_{r}(0), E_{1}(0), \ldots, E_{\nu_{0}-r}(0)\right\},
$$

say $E_{\nu_{0}-r}(0)$. Generalizing an argument in [10], define $h: \mathbf{R}^{n} \rightarrow M^{n}$ by

$$
\begin{aligned}
& h\left(t, x_{1}, \ldots, x_{r}, y_{1}, \ldots, y_{\nu_{0}-r-1}, u_{1}, \ldots, u_{n-v_{0}-r}, v_{1}, \ldots, v_{r}\right) \\
& \quad=\exp _{y(t)}\left(\sum x_{j} L_{j}(t)+\sum y_{k} E_{k}(t)+\sum u_{l} F_{l}(t)+\sum \hat{v}_{j} \hat{L}_{j}(t)\right) .
\end{aligned}
$$

Since

$$
\begin{aligned}
& \left(h_{*}\right)_{(t, 0)}(\partial / \partial t)=\vec{y}(t) \\
& \left(h_{*}\right)_{(t, 0)}\left(\partial / \partial x_{J}\right)=L_{j}(t) \\
& \left(h_{*}\right)_{(t, 0)}\left(\partial / \partial y_{k}\right)=E_{k}(t) \\
& \left(h_{*}\right)_{(t, 0)}\left(\partial / \partial u_{l}\right)=F_{l}(t) \\
& \left(h_{*}\right)_{(t, 0)}\left(\partial / \partial v_{j}\right)=\hat{L}_{j}(t)
\end{aligned}
$$

for each $t$, there is a neighborhood $U$ of $(t, \overrightarrow{0})$ such that $h$ is an imbedding on $U$. By shrinking, if necessary, we can find a neighborhood $V$ of $\{(t, \overrightarrow{0}) \mid t \in \mathbf{R}\}$ such that $h_{*}\left(\partial / \partial u_{l}\right)$ and $h_{*}\left(\partial / \partial v_{j}\right)$ are extensions of $F_{l}(t)$ and $\hat{L}_{j}(t)$ respectively to $h(V)$. By making $V$ smaller and restricting $t$ to $[a, b)$ we can assume $h_{*}\left(\partial / \partial u_{l}\right)$ and $h_{*}\left(\partial / \partial v_{\jmath}\right)$ span a complement in a neighborhood of $y(t), t \in[a, b)$.

In this neighborhood we let $Q$ be the projection defined by the decomposition $T_{x}(M)=T^{0}(x) \oplus T^{c}(x)$

$$
Q: T_{x}(M) \rightarrow T^{c}(x) .
$$

For any $Y \in T^{0}$ and $X \in T M$ we can define

$$
C_{Y} X=-Q\left(\nabla_{X} Y\right) \text {. }
$$

$C$ is called the conullity operator.

We need the following simple, technical lemma.

Lemma 1. Let $C$ and $Q$ be defined by (2.2) and (2.3). If $Y$ is in $T^{0}$ and $U$ and $V$ are in TM then

(1) $Q\left(\nabla_{Y} U\right)=Q\left(\nabla_{Y}(Q U)\right)$

(2) $Q\left(\nabla_{U-Q U} Y\right)=0$

(3) $\alpha(U, V)=\alpha(Q U, V)$

(4) $C$ is a tensor. 
Proof (1) Suppose $U=U_{0}+U_{c}$, where $U_{0} \in T^{0}$ and $U_{c} \in T^{c}$. Then $Q\left(\nabla_{Y} U\right)=Q\left(\nabla_{Y}\left(U_{0}+U_{c}\right)\right)=Q\left(\nabla_{Y} U_{c}\right)$, since $\nabla_{Y} U_{0}$ is in $T^{0}$. This means that $Q\left(\nabla_{Y} U\right)=Q\left(\nabla_{Y}(Q U)\right)$.

(2) If $U=U_{0}+U_{c}$ then $Q U=U_{c}$. This says that $U-Q U \in T^{0}$, so that $Q\left(\nabla_{U-Q U} Y\right)=0$.

(3) Again $\alpha(U, V)=\alpha\left(U_{0}+Q U, V\right)=\alpha(Q U, V)$.

(4) It is sufficient to show that $C_{\varphi Y}=\varphi C_{Y} U$ for $\varphi: M \rightarrow \mathbf{R}$.

$$
\begin{aligned}
C_{\varphi Y} U & =-Q\left(\nabla_{U} \varphi Y\right)=-Q\left[(U \varphi) Y+\varphi\left(\nabla_{U} Y\right)\right] \\
& =\varphi\left[-Q\left(\nabla_{U} Y\right)\right]=\varphi C_{Y} U .
\end{aligned}
$$

We now define a connection $\nabla^{\prime}$ in the complementary local bundle $T^{c}$. If $U \in T M, V \in T^{c}$, then

$$
\nabla_{U}^{\prime} V=Q\left(\nabla_{U} V\right)
$$

Using this connection we can differentiate $C$.

$$
\left(\nabla_{Y}^{\prime} C_{Y}\right) X=\nabla_{Y}^{\prime}\left(C_{Y} X\right)-C_{Y}\left(\nabla_{Y}^{\prime} X\right) .
$$

Another expression can be found for $\left(\nabla_{Y}^{\prime} C_{Y}\right) X$. The first term is

$$
\nabla_{Y}^{\prime}\left(C_{Y} X\right)=Q\left(\nabla_{Y}\left(C_{Y} X\right)\right)=-Q\left(\nabla_{Y}\left(Q\left(\nabla_{X} Y\right)\right)\right)=-Q\left(\nabla_{Y} \nabla_{X} Y\right) \text {. }
$$

The second term is, by Lemma (1.2),

$$
-C_{Y}\left(\nabla_{Y}^{\prime} X\right)=Q\left(\nabla_{\nabla_{Y}^{\prime} X} Y\right)=Q\left(\nabla_{Q\left(\nabla_{Y} X\right)} Y\right)=Q\left(\nabla_{\nabla_{Y} X} Y\right) .
$$

Combining both terms gives

$$
\nabla_{Y}^{\prime}\left(C_{Y} X\right)-C_{Y}\left(\nabla_{Y}^{\prime} X\right)=-Q\left(R(Y, X) Y+\nabla_{X} \nabla_{Y} Y-\nabla_{\nabla_{X} Y} Y\right) .
$$

If $W \in T^{1}$ then $\left\langle\nabla_{Y} Y, W\right\rangle=0$ and so $0=X\left\langle\nabla_{Y} Y, W\right\rangle=\left\langle\nabla_{X} \nabla_{Y} Y, W\right\rangle$ $+\left\langle\nabla_{Y} Y, \nabla_{X} W\right\rangle$. Along a geodesic $y_{t}$ in $T^{0}$ let $Y=\vec{y}_{t}$. Then $\nabla_{Y} Y=0$, and along $y_{t}$ we have $\left\langle\nabla_{X} \nabla_{Y} Y, W\right\rangle=0$ and $Q\left(\nabla_{X} \nabla_{Y} Y\right)=0$.

Next we claim that $Q\left(\nabla_{\nabla_{X} Y} Y\right)=C_{Y}\left(C_{Y} X\right)$. In fact $C_{Y}\left(C_{Y} X\right)=$ - $Q\left(\nabla_{C_{Y} X} Y\right)=Q\left(\nabla_{Q\left(\nabla_{X} Y\right)} Y\right)=Q\left(\nabla_{\nabla_{X} Y} Y\right)$ by Lemma 1 .

Finally then, if $Y$ is an extension of the tangent vectors $\vec{y}_{t}$ along a geodesic in $T^{0}$ then

$$
\left(\nabla_{Y}^{\prime} C_{Y}\right) X=Q(R(X, Y) Y)+C_{Y}^{2} X .
$$

THEOREM 2. If $f: M_{s}^{n} \rightarrow M_{t}^{n+k}(c)$ is an isometric immersion and $M^{n}$ is complete, then the relative nullity foliation is geodesically complete.

We first sketch a proof of Theorem 2 . 
Let $y_{t}$ be a geodesic in a leaf $L$ of $T^{0}$. It must be shown that $y_{t}$ can be extended indefinitely in $L$. Since $L$ is totally geodesic in $M$ we know that $y_{t}$ is a geodesic in $M$ which can be extended indefinitely in $M$ because $M$ is geodesically complete. It must be proven to lie entirely in $L$. Assume that $y_{t}$ is in $L$ for $t$ in $[a, b)$. If we can show that $y_{b}$ is in $G$, then we can take a coordinate system $\left\{y^{1}, \ldots, y^{n}\right\}$ adapted to the foliation with origin $y_{b}$, that is, with the property that the integral manifolds of $T^{0}$ are given by $y^{\nu_{0}+j}=c_{j}$. Now all points $y_{t}$, for $t$ less than and close to $b$, belong to one slice. As $t$ approaches $b, y_{t}$ approaches $y_{b}$ with coordinates $(0, \ldots, 0)$, so that $c_{1}, \ldots, c_{n-\nu_{0}}$ are all zero. Thus, $y_{b} \in L$ and we are done.

To show that $y_{b} \in G$ we need the following lemma, which will be proved after the proof of the theorem.

Lemma 2. For any $Z$ in $T_{y_{a}}(M)$ there exist $Z_{t} \in T_{y_{t}}(M), a \leq t<b$, such that $Z_{a}=Z$ and

$$
\nabla_{t}^{\prime}\left(Q Z_{t}\right)+C_{\vec{y}_{t}}\left(Q Z_{t}\right)=0 \text { for } a \leq t<b .
$$

Moreover, $Z_{t}$ can be extended differentiably to $t=b$. Here $\nabla_{t}^{\prime}$ stands for $\nabla_{\vec{y}_{i}}^{\prime}$.

The extension part of Lemma 2 will be proved using 2.6. Let $X_{t}$ be a parallel vector field along $y_{t}, a \leq t<b$, such that $X_{b} \in T^{0}\left(y_{b}\right)$. We will prove that $X_{a} \in T^{0}\left(y_{a}\right)$ so $\nu\left(y_{a}\right) \geq v\left(y_{b}\right) \geq \nu_{0}$ and $\nu\left(y_{b}\right)=\nu_{0}$. Take $Z_{t}$ as in Lemma 2. For each point $y_{t}, t<b$, extend $Y_{t}=\vec{y}_{t}, X_{t}$ and $Z_{t}$ to vector fields $Y, X$ and $Z$ with $Y$ in $T^{0}$.

Examine Codazzi's equation with $Y, X$ and $Z$.

$$
\begin{aligned}
& \nabla_{Y}^{\perp} \alpha(Z, X)-\alpha\left(\nabla_{Y} Z, X\right)-\alpha\left(Z, \nabla_{Y} X\right) \\
& =\nabla_{Z}^{\perp} \alpha(Y, X)-\alpha\left(\nabla_{Z} Y, X\right)-\alpha\left(Y, \nabla_{Z} X\right)
\end{aligned}
$$

Along $y_{t} \nabla_{Y} X=0$ and $\alpha(Y, X)=0$ and $\alpha\left(Y, \nabla_{Z} X\right)=0$. The equation reduces to

$$
\nabla_{Y}^{\perp} \alpha(Z, X)-\alpha\left(\nabla_{Y} Z, X\right)=-\alpha\left(\nabla_{Z} Y, X\right)=\alpha\left(C_{Y} Z, X\right)
$$

by Lemma (1.3). This gives

along $y_{t}$.

$$
\begin{aligned}
\nabla_{Y}^{\perp} \alpha(Z, X) & =\alpha\left(\nabla_{Y} Z, X\right)+\alpha\left(C_{Y} Z, X\right) \\
& =\alpha\left(Q\left(\nabla_{Y}(Q Z)\right), X\right)+\alpha\left(C_{Y} Z, X\right) \\
& =\alpha\left(\nabla_{t}^{\prime} Q Z, X\right)+\alpha\left(C_{\vec{y}_{t}} Z, X\right)
\end{aligned}
$$

This is true for $t<b$, so by continuity it holds for $t=b$. That is to say that $\alpha(Z, X)$ is parallel along $y_{t}, a \leq t \leq b . \alpha(Z, X)=0$ at $t=b$ 
which means $\alpha(Z, X)=0$ at $y_{a}$. Since $Z_{a}$ is arbitrary, $X_{a} \in T^{0}\left(y_{a}\right)$, as desired.

Proof of Lemma 2. In what follows we use the same notation that was introduced in the definition of $T^{c}(x)$. Along $y_{t}$ write

$$
Q Z_{t}=\sum \varphi_{j}(t) \hat{L}_{j}(t)+\sum \psi_{p}(t) F_{p}(t)
$$

We want to solve $Q\left(\nabla_{t} Q Z_{t}\right)+C_{\vec{y}_{t}}\left(Q Z_{t}\right)=0$. Rewriting, this is

$$
Q\left(\nabla_{t}\left(\sum \varphi_{j}(t) \hat{L}_{j}(t)+\sum \psi_{p}(t) F_{p}(t)\right)\right)+C_{\vec{y}_{t}}\left(Q Z_{t}\right)=0
$$

or

$$
Q\left(\sum \varphi_{j}^{\prime}(t) \hat{L}_{j}(t)+\sum \psi_{p}^{\prime}(t) F_{p}(t)\right)+C_{\vec{y}_{t}}\left(Q Z_{t}\right)=0
$$

or

$$
\sum \varphi_{j}^{\prime}(t) \hat{L}_{J}(t)+\sum \psi_{p}^{\prime}(t) F_{p}(t)+C_{\vec{y}_{t}}\left(Q Z_{t}\right)=0 .
$$

Since $C_{\vec{y}_{t}}\left(Q Z_{t}\right)$ can be written in terms of $\hat{L}_{j}(t)$ and $F_{p}(t)$ this is a system of ordinary differential equations which can be solved for $a \leq t<b$. To see that the solution can be extended: By (2.6)

$$
\left(\nabla_{Y}^{\prime} C_{Y}\right) X=C_{Y}^{2} X+Q(R(X, Y) Y) \text { for all } X \text { in } T M \text {. }
$$

$Q(R(X, Y) Y)=c\langle Y, Y\rangle Q X$, so that along $y_{t}, a \leq t<b$,

$$
\left(\nabla_{Y}^{\prime} C_{Y}\right)=C_{Y}^{2}+c\langle Y, Y\rangle Q \text {. }
$$

If $Z_{t}$ satisfies (2.7), then by differentiating once more we have:

$$
\nabla_{t}^{\prime 2}\left(Q Z_{t}\right)+\nabla_{t}^{\prime} C_{\vec{y}_{t}}\left(Q Z_{t}\right)=0
$$

or

$$
\nabla_{t}^{\prime 2}\left(Q Z_{t}\right)+\left(\nabla_{t}^{\prime} C_{\vec{\gamma}_{t}}\right) Q Z_{t}+C_{Y}\left(\nabla_{t}^{\prime}\left(Q Z_{t}\right)\right)=0 .
$$

This yields, using (2.8),

$$
\nabla_{t}^{\prime 2}\left(Q Z_{t}\right)+C_{Y}^{2}\left(Q Z_{t}\right)+c\langle Y, Y\rangle Q Z_{t}+C_{Y}\left(\nabla_{t}^{\prime}\left(Q Z_{t}\right)\right)=0
$$

Plugging in (2.7) gives

$$
\nabla_{t}^{\prime 2}\left(Q Z_{t}\right)+C_{Y}^{2}\left(Q Z_{t}\right)+c\langle Y, Y\rangle Q Z_{t}-C_{Y}^{2}\left(Q Z_{t}\right)=0,
$$

i.e.,

$$
\nabla_{t}^{\prime}\left(Q Z_{t}\right)-c\langle Y, Y\rangle Q Z_{t}=0 \text { for } a \leq t<b .
$$

In terms of the parallel basis for $T^{c}$ along $y_{t}(2.9)$ can be written

$$
\frac{d^{2} \varphi_{j}(t)}{d t^{2}}+c\langle Y, Y\rangle \varphi_{j}=0, \quad \frac{d^{2} \psi_{p}(t)}{d t^{2}}+c\langle Y, Y\rangle \psi_{p}=0 .
$$

Solutions to these equations can be extended differentiably beyond $b$. 
To see that (2.6) is an equation of Ricattti-type write

$$
\begin{aligned}
& C_{Y} \hat{L}_{j}(t)=\sum_{i=1}^{r} C_{i j}^{L}(t) \hat{L}_{i}(t)+\sum_{p=1}^{n-\nu_{0}-r} C_{p j}^{F}(t) F_{p}(t) \\
& C_{Y} F_{q}(t)=\sum_{i=1}^{r} D_{i q}^{L}(t) \hat{L}_{i}(t)+\sum_{p=1}^{n-\nu_{0}-r} D_{p q}^{F}(t) F_{p}(t) .
\end{aligned}
$$

Then

$$
\begin{gathered}
\left(\nabla_{Y}^{\prime} C_{Y}\right) \hat{L}_{j}(t)=\nabla_{Y}^{\prime}\left(C_{Y} \hat{L}_{j}(t)\right)-C_{Y}\left(\nabla_{Y}^{\prime} \hat{L}_{j}(t)\right) \\
=\nabla_{Y}^{\prime}\left[\sum_{i} C_{i j}^{L}(t) \hat{L}_{i}(t)+\sum_{p} C_{p j}^{F}(t) F_{p}(t)\right] \\
=\sum_{i} \frac{d C_{i j}^{L}(t)}{d t} \hat{L}_{i}(t)+\sum_{p} \frac{d C_{p j}^{F}}{d t} F_{p}(t) .
\end{gathered}
$$

Similarly,

$$
\begin{gathered}
\left(\nabla_{Y}^{\prime} C_{Y}\right) F_{q}(t)=\sum_{i} \frac{d D_{i q}^{L}(t)}{d t} \hat{L}_{i}(t)+\sum_{p} \frac{d D_{p q}^{F}(t)}{d t} F_{p}(t) \\
Q\left(R\left(\hat{L}_{j}(t), Y\right) Y\right)=c\langle Y, Y\rangle \hat{L}_{j}(t) \\
Q\left(R\left(F_{q}(t), Y\right) Y\right)=c\langle Y, Y\rangle F_{q}(t)
\end{gathered}
$$

Let

$$
C(t)=\left[\begin{array}{ll}
C_{i j}^{L}(t) & D_{i q}^{L}(t) \\
C_{p j}^{F}(t) & D_{p q}^{F}(t)
\end{array}\right] \text { and } K(t)=c\langle Y, Y\rangle I_{n} .
$$

Then, (2.6) can be written as

$$
\frac{d C(t)}{d t}=C^{2}(t)+K(t)
$$

3. Applications of the Ricatti-type differential equation. Let $M_{1}^{n}$ be a geodesically complete Lorentzian submanifold of $\tilde{M}_{1}^{n+k}(c)(c>0)$, where $\tilde{M}_{1}^{n+k}(c)$ is the Lorentzian space form of constant curvature $c$. Let $\nu_{0}$ be the index of relative nullity of $M_{1}^{n}$ in $\tilde{M}_{1}^{n+k}(c)$.

It is well-known that for $K(t)>0$, the equation (2.10) has no global solution with an initial condition $C(0)$ which has a real eigenvalue. This implies, in our case, that there is no global solution of (2.10) if $\langle Y, Y\rangle>0$. If $\langle Y, Y\rangle=0,0$ is the only global solution under an initial condition $C(0)$ 
with a real eigenvalue. In particular, if $C(t)$ has a real eigenvalue for some $t, Y$ must be 0 , provided that $Y$ is space-like.

Here let us state an elementary fact on a Lorentzian vector space.

Proposition 3. Let $L^{n}$ be a Lorentzian inner product space with the inner product $\langle$,$\rangle . Let V^{\nu}$ be a linear subspace of dimension $\nu$. Then,

(i) $V^{\nu}=L^{\nu}$ and $\left.\langle\rangle\right|_{V^{\nu}}$ is non-degenerate, or

(ii) $V^{\nu}=E^{\nu}$ and $\left.\langle\rangle\right|_{V^{\nu}}$ is positive definite, or

(iii) $\left.\langle\rangle\right|_{,V^{\nu}}$ is degenerate and $V^{\nu}=E^{\nu-1}+\operatorname{span}\{\xi\}$, where $\langle\xi, \xi\rangle=0$ and $\zeta \perp E^{\nu-1}$.

\section{(Proof). See Graves [9].}

Now let $Y_{1}, \ldots, Y_{\nu_{0}-1}$ be a set of $\left(\nu_{0}-1\right)$, linearly independent space-like vectors in $T^{0}(x)$ such that $\operatorname{span}\left\{Y_{1}, \ldots, Y_{\nu_{0}-1}\right\}$ is a positive definite subspace of $T^{0}(x)$. Proposition 1 tells us the choice of $Y_{1}, \ldots, Y_{\nu_{0}-1}$ is possible. Denote $C_{t}=C_{Y_{t}}\left(i=1, \ldots, \nu_{0}-1\right)$ for simplicity.

Lemma 3. The set of vectors $\left\{X, C_{1}(X), \ldots, C_{\nu_{0}-1}(X)\right\}$ forms a $\nu_{0}$-frame in $T^{c}(x)$ for $X \neq 0 \in T^{c}(x)$.

$$
\begin{aligned}
& \text { Proof. Let } \alpha X+\alpha_{1} C_{1}(X)+\cdots+\alpha_{\nu_{0}-1} C_{\nu_{0}-1}(X)=0 \text {. Then } \\
& \alpha_{1} C_{1}(X)+\cdots+\alpha_{\nu_{0}-1} C_{\nu_{0}-1}(X)=C_{\alpha_{1} Y_{1}+\cdots+\alpha_{\nu_{0}-1} Y_{\nu_{0}-1}}(X)=(-\alpha) X .
\end{aligned}
$$

Hence, $-\alpha$ is a real eigenvalue of $C_{\alpha_{1} Y_{1}+\cdots+\alpha_{\nu_{0}-1} Y_{\nu_{0}-1}}$. Since $\alpha_{1} Y_{1}$ $+\cdots+\alpha_{v_{0}-1} Y_{\nu_{0}-1}$ is a space-like vector, $\alpha_{1} Y_{1}+\cdots+\alpha_{\nu_{0}-1} Y_{\nu_{0}-1}=0$ from the above remark. This implies $\alpha_{1}=\cdots=\alpha_{\nu_{0}-1}=0$ and $\alpha=0$.

As was done in [7], denote by $V_{n, r}$ the Stiefel manifold of ordered $r$-frames in $E^{n}$. It is well known that $V_{n, r} \rightarrow V_{n, 1}$ is a principal fiber bundle in a natural way. Denote by $\rho(n)$ the largest integer such that the fibration $V_{n, \rho(n)} \rightarrow V_{n, 1}$ has a global cross-section. Define by $v_{n}$ the largest integer such that $\rho\left(n-\nu_{n}\right) \geq \nu_{n}$.

THEOREM 3. Let $M_{1}^{n}$ be a geodesically complete, connected submanifold of $\tilde{M}_{1}^{n+k}(c), c>0$. If the index of relative nullity $\nu_{0}>\nu_{n}$, then $M_{1}^{n}$ is totally geodesic in $\tilde{M}_{1}^{n+k}(c)$ and $\nu_{0}=n$.

Proof. For any $x \in G, T^{0}(x)$ always contains a copy of $E^{\nu_{0}-1}$. By Lemma $3, V_{n-\nu_{0}, \nu_{0}} \rightarrow V_{n-\nu_{0}, 1}$ has a global cross-section $\varphi$ defined by $\varphi(X)=\left(X, C_{1} X, \ldots, C_{\nu_{0}-1} X\right)$ for $\forall X \neq 0 \in T^{c}(x)$. Hence, $\rho\left(n-\nu_{0}\right) \geq$ $\nu_{0}$; therefore, $\nu_{0} \leq \nu_{n}$. 
REMARK. If one of the relative nullity leaves is a Riemannian manifold relative to the induced metric, the above $\nu_{n}$ can be improved by defining $\bar{\nu}_{n}$ to be the largest integer such that $\rho\left(n-\bar{\nu}_{n}\right) \geq \bar{\nu}_{n}+1$. Clearly $\bar{\nu}_{n} \leq \nu_{n}$.

Some of the numerical values for $\nu_{n}$ are as follows: $\nu_{1}=0, \nu_{2}=1$, $\nu_{3}=1, \nu_{4}=2, \nu_{5}=1, \nu_{6}=2, \nu_{7}=3, \nu_{8}=4, \nu_{9}=1$.

The argument used here can be applied to obtain a similar result for more general indefinite metrics. Unlike the Riemannian case, it is known that the above $\nu_{n}$ 's are often the best possible value. For example, Graves and Nomizu [11] constructed an isometric immersion of $S_{1}^{2}$ into $S_{1}^{3}$ with the index of relative nullity 1 .

Our next result states:

THEOREM 4. Let $f: M_{1}^{n} \rightarrow \tilde{M}_{1}^{n+p}(c)$ be an isometric immersion between two Lorentzian manifolds, where $\tilde{M}_{1}^{n+p}(c)$ is the Lorentzian space form of positive curvature c. Suppose that the Ricci curvature $S$ of $M_{1}^{n}$ satisfies $S(X, X) \geq(n-1) c\langle X, X\rangle$ for all space-like vectors $X$.

(1) If $T^{0}(x)$ is Lorentzian for some $x \in G$, then the index of relative nullity is either 0 or $n$.

(2) If $T^{0}(x)$ is degenerate for some $x \in G$, then the index of relative nullity is $0,1, n-1$ or $n$.

(3) If $T^{0}(x)$ is Riemannian for all $x \in G$ and if $p=1$, then the index of relative nullity is $0, n-2, n-1$, or $n$.

We will prove Theorem 4 after a sequence of propositions and lemmas.

Proposition 4. Let (, ) be a symmetric bilinear form on an $n-\nu_{0}$ dimensional vector space $V$ over $\mathbf{R}$ with signature $\left(m_{1}, m_{2}, m_{3}\right)$. If $m_{1} \neq m_{2}$ and $T: V \rightarrow V$ is a symmetric linear operator with respect to $($,$) , then T$ has a real eigenvalue. Note a symmetric bilinear form of signature $\left(m_{1}, m_{2}, m_{3}\right)$ has $m_{1}(-1)$ 's, $m_{2}(+1)$ 's and $m_{3} 0$ 's in the canonical form.

Proof. Choose a canonical basis $\left\{e_{1}, \ldots, e_{m_{1}}, f_{1}, \ldots, f_{m_{2}}, g_{1}, \ldots, g_{m_{3}}\right\}$ of $V$ for the symmetric bilinear form, so that $\left(e_{\alpha}, e_{\alpha^{\prime}}\right)=-\delta_{\alpha \alpha^{\prime}},\left(f_{\beta}, f_{\beta^{\prime}}\right)=$ $\delta_{\beta \beta^{\prime}},\left(g_{\gamma}, g_{\gamma^{\prime}}\right)=0$ and all other products are zero. The matrix of the 
symmetric operator $T$ with respect to this basis has the form:

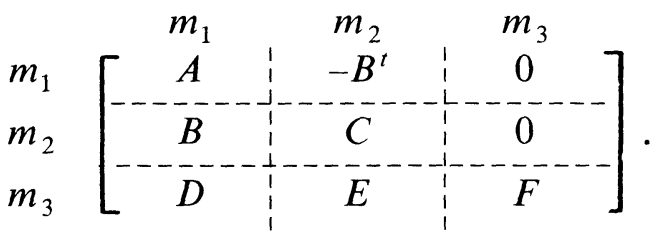

Here $A^{t}=A$ and $C^{t}=C$. Denote this matrix by $M_{T}$. The matrix $M_{s}=$ $\left[\begin{array}{cc}A & -B^{t} \\ B & C\end{array}\right]$ represents a symmetric linear transformation on the span of $\left\{e_{1}, \ldots, e_{m_{1}}, f_{1}, \ldots, f_{m_{2}}\right\}$. By a change of basis, $M_{s}$ can be put into a standard form [15]. If $m_{1} \neq m_{2}$, there is one block of the form:

$$
\left[\begin{array}{cccc}
\lambda & 1 & \cdots & 0 \\
\vdots & & \ddots & \vdots \\
0 & & \cdots & \lambda
\end{array}\right] .
$$

Thus, $M_{s}$ has an eigenvalue $\lambda$. Noting that

$$
\operatorname{det}\left[\lambda I-M_{T}\right]=\operatorname{det}\left[\lambda I-M_{s}\right] \operatorname{det}[\lambda I-F]=0,
$$

we see that $T$ has a real eigenvalue $\lambda$.

Now let $\eta=\sum_{i=1}^{n}\left\langle X_{l}, X_{l}\right\rangle \alpha\left(X_{l}, X_{l}\right)$, where $\left\{X_{1}, \ldots, X_{n}\right\}$ is an orthonormal basis of $T_{x}(M)$. Define a bilinear form $():, T^{c}(x) \times T^{c}(x) \rightarrow$ $\mathbf{R}$ by, for any $X$ and $Y \in T^{c}(x)$,

$$
(X, Y)=\langle\alpha(X, Y), \eta\rangle .
$$

It is clear that (, ) is symmetric, since $\alpha$ is symmetric. We may find an alternative expression for $($, ) using the Gauss equation and the Ricci curvature of $M_{1}^{n}$.

$$
\begin{aligned}
(X, Y)= & \langle\alpha(X, Y), \eta\rangle \\
= & \sum_{i=1}^{n}\left\langle X_{i}, X_{i}\right\rangle\left\langle\alpha(X, Y), \alpha\left(X_{\imath}, X_{\imath}\right)\right\rangle \\
= & S(X, Y)-n c\langle X, Y\rangle+c\langle X, Y\rangle \\
& +\sum_{i=1}^{n}\left\langle X_{i}, X_{i}\right\rangle\left\langle\alpha\left(X_{i}, X\right), \alpha\left(X_{i}, Y\right)\right\rangle .
\end{aligned}
$$

Clearly, the definition of $($,$) and the above expression for (X, Y)$ do not depend on the choice of the orthonormal basis $\left\{X_{1}, \ldots, X_{n}\right\}$. 
If $\left\{\xi_{1}, \ldots, \xi_{p}\right\}$ is an orthonormal basis for $N(x)$, then the last term of the above expression can be rewritten

$$
\begin{aligned}
& \sum_{i, j}\left\langle X_{i}, X_{i}\right\rangle\left\langle\xi_{j}, \xi_{j}\right\rangle\left\langle\alpha\left(X_{i}, X\right), \xi_{j}\right\rangle\left\langle\alpha\left(X_{i}, Y\right), \xi_{j}\right\rangle \\
& =\sum_{i, j}\left\langle X_{i}, X_{i}\right\rangle\left\langle\xi_{j}, \xi_{j}\right\rangle\left\langle A_{\xi_{j}} X, X_{i}\right\rangle\left\langle A_{\xi_{j}} Y, X_{i}\right\rangle \\
& =\sum_{j}\left\langle\xi_{j}, \xi_{j}\right\rangle\left\langle A_{\xi_{j}} X, A_{\xi_{j}} Y\right\rangle .
\end{aligned}
$$

Since the normal space is positive definite,

$$
(X, Y)=S(X, Y)-c(n-1)\langle X, Y\rangle+\sum_{j=1}^{p}\left\langle A_{\xi_{j}}^{2} X, Y\right\rangle .
$$

For convenience, let $\tau=c(n-1)$, and $A_{\xi}$, by $A_{j}$. We examine (, ) by looking at

$$
h(X, Y):=S(X, Y)-\tau\langle X, Y\rangle \text { and } k(X, Y):=\sum_{j=1}^{p}\left\langle A_{J}^{2} X, Y\right\rangle .
$$

LEMMA 4. If the Ricci curvature satisfies the hypothesis in the theorem, then $h(X, Y)$ is positive semi-definite or Lorentzian.

Proof. We show that $h$ cannot have two (-1)'s in its signature. If it did, we could find a pair of linearly independent vectors $e$ and $f$ in $T_{x} M$ such that $h(e, e)=-1, h(f, f)=-1$ and $h(e, f)=0$ i.e., $S(e, e)=-1$ $+\tau\langle e, e\rangle, \quad S(f, f)=-1+\tau\langle f, f\rangle$ and $S(e, f)=\tau\langle e, f\rangle$. By the hypothesis, it would then be the case that $\langle e, e\rangle \leq 0$ and $\langle f, f\rangle \leq 0$.

We now examine the various possibilities for the lengths of $e$ and $f$. In each case, we will find a space-like vector which violates the condition on the Ricci curvature. We will use the reverse Cauchy-Schwarz inequality, i.e, $0 \leq\langle e, e\rangle\langle f, f\rangle \leq\langle e, f\rangle^{2}$ in this case. In the following argument, denote $\langle e, f\rangle$ by $a$ for brevity. $\langle e, f\rangle=a$ can be positive.

If both $e$ and $f$ were light-like, $e+f$ would be space-like and $\langle e+f, e+f\rangle=2 a$.

$$
\begin{aligned}
S(e+f, e+f) & =S(e, e)+2 S(e, f)+S(f, f) \\
& =(-1)+2 \tau\langle e, f\rangle+(-1)<2 \tau\langle e, f\rangle=2 \tau a \\
& =\tau\langle e+f, e+f\rangle .
\end{aligned}
$$

This contradicts the hypothesis. 
If $e$ were light-like and $f$ were time-like, we could also assume that $\langle f, f\rangle=-1$. Then $e+a f$ would be space-like, for $\langle e+a f, e+a f\rangle=a^{2}$. But

$$
\begin{aligned}
S(e+a f, e+a f) & =S(e, e)+2 a S(e, f)+a^{2} S\langle f, f\rangle \\
& =-1+2 a \tau a+a^{2}(-1-\tau)<\tau a^{2},
\end{aligned}
$$

a contradiction.

If $e$ and $f$ are both time-like, we could assume that $\langle e, e\rangle=-1=$ $\langle f, f\rangle$. Then, $e+a f$ is space-like with $\langle e+a f, e+a f\rangle=-1+a^{2}>0$ by the reverse Cauchy-Schwarz inequality.

$$
S(e+a f, e+a f)=-1-\tau+2 a^{2} \tau-a^{2}-a^{2} \tau<\left(-1+a^{2}\right) \tau,
$$

a contradiction.

Next we show that if there are any vectors which are time-like with respect to $h$, then $h$ is non-degenerate and therefore Lorentzian. If this is not the case, there are linearly independent $e$ and $g$ such that $h(e, e)=-1$, $h(g, g)=0$ and $h(e, g)=0$, i.e., $S(e, e)=-1+\tau\langle e, e\rangle, \quad S(g, g)=$ $\tau\langle g, g\rangle$ and $S(e, g)=\tau\langle e, g\rangle$. We know that $\langle e, e\rangle \leq 0$ and can assume that $\langle e, g\rangle \geq 0$. If $\langle g, g\rangle>0$, then $\langle g+e, g+e\rangle=\langle g, g\rangle+2\langle e, g\rangle$ $>0$, so that $g+e$ is space-like. However,

$$
\begin{aligned}
S(g+e, g+e)= & \tau\langle g, g\rangle+2 \tau\langle e, g\rangle-1 \\
& +\tau\langle e, e\rangle\langle\tau[\langle g, g\rangle+2\langle e, g\rangle],
\end{aligned}
$$

a contradiction. The only remaining possibility is that $\langle e, e\rangle \leq 0$ and $\langle g, g\rangle \leq 0$. The span of $e$ and $g$ is non-degenerate, so for some $k \in \mathbf{R}$, $e+k g$ is space-like.

$$
\begin{aligned}
S(e+k g, e+k g) & =S(e, e)+2 k S(e, g)+k^{2} S(g, g) \\
& =-1+\tau\langle e, e\rangle+2 k \tau\langle e, g\rangle+k^{2} \tau\langle g, g\rangle \\
& <\tau\left[\langle e, e\rangle-2 k\langle e, g\rangle+k^{2}\langle g, g\rangle\right],
\end{aligned}
$$

a contradiction.

This completes the proof of Lemma 4.

We now turn to $k(X, Y)=\sum_{j=1}^{p}\left\langle A_{j}^{2} X, Y\right\rangle$ on $T^{c}(x)$.

Lemma 5. If $T^{c}(x)$ is positive definite, then $k$ restricted to $T^{c}(x)$ is positive definite.

Proof.

$$
k(X, X)=\sum_{j=1}^{p}\left\langle A_{j}^{2} X, X\right\rangle=\sum_{j=1}^{p}\left\langle A_{j} X, A, X\right\rangle .
$$


If $k(X, X)=0$, then $A_{j} X=0$ for $j=1, \ldots, p$, since $T^{c}(x)=T^{1}(x)=$ $\operatorname{span}\left\{A_{j} Y\right\}$ is positive definite. This means that $X \in T^{0}(x) \cap T^{1}(x)$; therefore, $X=0$. Hence, $k$ is positive definite.

Proposition 5. If $T^{c}(x)$ is positive definite, then (, ) is positive definite on $T^{c}(x)$.

Proof. For no non-zero $e \in T^{c}(x)$ is $h(e, e)=-1$, since this implies $\langle e, e\rangle \leq 0$. Thus, the form ( , ) is the sum of a positive semi-definite form and a positive definite form.

Lemma 6. If $T^{c}(x)$ is a degenerate subspace with respect to $\langle$,$\rangle , then h$ is positive semi-definite on $T^{c}(x)$ for $\operatorname{dim} T^{c}(x)>1$.

Proof. $T^{c}(x)$ is a positive semi-definite subspace with respect to $\langle$, by hypothesis. If there were an $e \in T^{c}(x)$ with $h(e, e)=-1$, then $\langle e, e\rangle$ $=0$. By Lemma 4, there would be $g \in T^{c}(x)$ such that $h(g, g)=1$ and $h(e, g)=0$. By hypothesis we would have $\langle g, g\rangle>0$ and $\langle e, g\rangle=0$. For all $t \in \mathbf{R}\langle g+t e, g+t e\rangle=\langle g, g\rangle>0$, but

$$
S(g+t e, g+t e)=1+\tau\langle g, g\rangle-t^{2}<\tau\langle g, g\rangle .
$$

This is a contradiction.

LEMMA 7. If $T^{c}(x)$ is a degenerate subspace with respect to $\langle$,$\rangle and if$ $\operatorname{dim} T^{c}(x)>1$, then $k$ is positive semi-definite on $T^{c}(x)$ and for some $Y \in T^{c}(x) k(Y, Y)>0$.

Proof. If $T^{0}(x)$ is degenerate, so is $T^{1}(x)=\operatorname{span}\left[A_{\xi} Y\right]$. Thus, there is a light-like vector $L$ such that, for any normal vector $\xi, \operatorname{Im} A_{\xi} \subseteq[L]^{\perp}$. The metric on $[L]^{\perp}$ is positive semi-definite, so $\left\langle A_{\xi} Y, A_{\xi} Y\right\rangle \geq 0$ for all $\xi$, $Y$. This implies that $k$ is positive semi-definite. If $k(Y, Y)=0$ for all $Y$, then each $A_{\xi} Y$ is light-like. We also have $\left\langle A_{\xi} Y, L\right\rangle=0$ for $\xi, Y$. Recalling that perpendicular light-like vectors are linearly dependent, we see that $T^{1}(x)$ would be one-dimensional, which is not the case.

Proposition 6. If $T^{c}(x)$ is a degenerate subspace, then (, ) is positive semi-definite on $T^{c}(x)$ and for some $Y \in T^{c}(x),(Y, Y)>0$.

Finally, we assume $M_{1}^{n}$ is a hypersurface in $\tilde{M}_{1}^{n+1}$ and that $T^{c}=T^{1}$ is Lorentzian. Here $k(X, Y)=\langle A X, A Y\rangle$ where $A$ is a transformation of $T^{1}$ which is one-to-one. 
Proposition 7. If $M_{1}^{n}$ is a hypersurface in $\tilde{M}_{1}^{n+1}(c), T^{1}$ is Lorentzian and $n-\nu_{0}>2$, then the signature $\left(m_{1}, m_{2}, m_{3}\right)$ of $($,$) has m_{2}>m_{1}$.

Proof. We know that $h$ is positive semi-definite or Lorentzian. The shape operator $A$ can be put into one of four canonical forms [15] and $k$ can be explicitly calculated using these forms.

$$
A=\left[\begin{array}{rrrrr}
\lambda & 0 & & \\
1 & \lambda & & & \\
& & \lambda_{1} & \\
& & & \ddots & \\
& & & \lambda_{n-\nu_{0}-2}
\end{array}\right],
$$

where $A$ is given with respect to a pseudo-orthonormal basis $\left\{\hat{L}, L, f_{1}, \ldots, f_{n-\nu_{0}-2}\right\} . k$ then has the following matrix with respect to this basis:

$$
\left[\begin{array}{rrrl}
-2 \lambda & -\lambda^{2} & & \\
-\lambda^{2} & 0 & & \\
& & \lambda_{1}^{2} & \\
& & \ddots & \lambda_{n-\nu_{0}-2}^{2}
\end{array}\right] .
$$

Note that $\lambda \lambda_{1} \cdots \lambda_{n-\nu_{0}-2} \neq 0$ and the signature of $k$ is $(1, n-$ $\left.\nu_{0}-1\right)$. We can find a space-like vector $U$ in $\operatorname{span}\{\hat{L}, L\}$ such that $k(U, U)>0$. In fact, there are choices for $b \in \mathbf{R}$ such that $\langle\hat{L}+b L$, $\hat{L}+b L\rangle=-2 b$ and $k(\hat{L}+b L, \hat{L}+b L)=-2 \lambda(1+b \lambda)$ are both positive. Set $U=\hat{L}+b L$. We claim that $($,$) is positive definite on$ $\operatorname{span}\left\{U, f_{1}, \ldots, f_{n-\nu_{0}-2}\right\}$.

$$
h\left(c U+\sum c_{j} f_{j}, c U+\sum c_{j} f_{j}\right) \geq 0
$$

since

$$
\left\langle c U+\sum c_{j} f_{j}, c U+\sum c_{j} f_{j}\right\rangle=c^{2}\langle U, U\rangle+\sum c_{j}^{2} \geq 0 .
$$

It is also clear that $k\left(c U+\sum c_{j} f_{j}, c U+c_{j} f_{j}\right) \geq 0$ and is equal to 0 if and only if $c=0=c_{j}$ for $j=1, \ldots, n-\nu_{0}-2$. Hence, if $n-\nu_{0}>2$, the signature of $($, ) will have more plus signs than minus signs.

(ii)

$$
A=\left[\begin{array}{rrrrl}
\lambda & 0 & 0 & & \\
0 & \lambda & 1 & & \\
-1 & 0 & \lambda & & \\
& & & \lambda_{1} & \\
& & & \ddots & \lambda_{n-\nu_{0}-3}
\end{array}\right]
$$


with respect to a pseudo-orthonormal basis $\left\{\hat{L}, L, f, f_{1}, \ldots, f_{n-\nu_{0}-3}\right\} . k$ has the following form with respect to the above basis:

$$
\left[\begin{array}{rrrrrr}
1 & -\lambda^{2} & -2 \lambda & & & \\
-\lambda^{2} & 0 & 0 & & & \\
-2 \lambda & 0 & \lambda^{2} & & & \\
& & & \lambda_{1}^{2} & & \\
& & & & \ddots & \\
& & & & & \lambda_{n-\nu_{0}-3}^{2}
\end{array}\right]
$$

In this case, we construct a space-like vector $U=\hat{L}+b L$ such that $k(U, U)>4$. That is, we want $\langle\hat{L}+b L, \hat{L}+b L\rangle=-2 b>0$ and $k(\hat{L}+b L, \hat{L}+b L)=1-2 b \lambda^{2}>4$.

Here, too, $\langle$,$\rangle is positive definite on \operatorname{span}\left\{U, f, f_{1}, \ldots, f_{n-\nu_{0}-3}\right\}$. For any vector $V$ in this span, $\langle V, V\rangle \geq 0$. Therefore, $h(V, V) \geq 0$ and the fact that $k(U, U)>4$ guarantees that $k(V, V) \geq 0$ with $k(V, V)=0$ if and only if $V=0$.

$$
A=\left[\begin{array}{rrrrl}
a & -b & & & \\
b & a & & & \\
& & \lambda_{1} & & \\
& & & \ddots & \lambda_{n-\nu_{0}-2}
\end{array}\right]
$$

with respect to orthonormal basis $\left\{e, f, f_{1}, \ldots, f_{n-\nu_{0}-2}\right\}$. We can find a space-like vector $U$ in $\operatorname{span}\{e, f\}$ with $k(U, U)>0$. As above, the signature of ( , ) has more plus signs than minus signs.

(iv) $A$ is diagonalizable with respect to an orthonormal basis. Then, it is easy to see that the conclusion of the proposition is satisfied. This completes the proof.

We are now in the position of proving Theorem 4 . We follow the argument in [7]. If $n>\nu_{0}>0$, choose any non-zero $Z \in T^{0}(x)$. Then, $C_{Z}: T^{c}(x) \rightarrow T^{c}(x)$ is a symmetric operator with respect to $($,$) . In (1), by$ Propositions 4 and 5, $C_{Z}$ would have a real eigenvalue. Also $Z$ can be chosen as a space-like vector. But the equation (2.10) has no global solution in which $k(t)>0$ and $C_{Z}$ has a real eigenvalue. This is a contradiction. In case (2), if $n-\nu_{0}>1$, by Propositions 4 and $6, C_{Z}$ would have a real eigenvalue. Clearly, $Z$ can be chosen to be a space-like vector; hence, a contradiction as before. Finally, in case (3), Propositions 4 and 7 assure us a real eigenvalue of $C_{Z}$. Since $n-\nu_{0}>2$ and since $T^{1}$ is Lorentzian, $Z$ can be chosen to be space-like. The same argument as above completes the proof. 
The following result concerns the over simplified "The axiom of sphere" for the hypersurfaces in the Lorentzian space form of positive constant curvature $c$.

Let $f: M_{1}^{n} \rightarrow \tilde{M}_{1}^{n+1}(c)$ be a geodesically complete Lorentzian hypersurface, where $\tilde{M}_{1}^{n+1}(c)$ is the Lorentzian space form of positive curvature $c$. Let us assume:

(*) Through each point $x$ of $M$ exists a $k(x)$-dimensional local submanifold $S_{x}$ of $M$ which is mapped under $f$ isometrically into a $k(x)$-dimensional totally geodesic submanifold of $\tilde{M}_{1}^{n+1}(c)$.

TheOREM 5. If $2 k(x)-n \geq \nu_{n}, M_{1}^{n}$ is totally geodesic in $\tilde{M}_{1}^{n+1}(c)$. Here $\nu_{n}$ is the numerical value determined in Theorem 3.

LEMMA 8. If $2 k(x)-n>0$, the relative nullity $\nu(x)$ at $x \geq 2 k(x)-$ $n$. In particular, the index of relative nullity $\nu_{0} \geq 2 k-n$, where $k=$ $\min _{x \in M_{1}^{n}} k(x)$.

Proof. If $S_{x}$ is non-degenerate, the result of Lemma 8 will be obtained in the same manner as in [3]. Now let us assume that $S_{x}$ is degenerate. Let $\hat{e}_{1}(x), \ldots, \hat{e}_{n-k-1}(x), L$ form a pseudo-orthonormal basis for $\left(T S_{x}\right)^{\perp}$, which is the orthogonal complement of $T S_{x}$ in $T_{x} M_{1}^{n}$. Extend it to a basis of $T_{x} M$ by adding $\left\{e_{1}(x), \ldots, e_{k-1}(x), \hat{L}(x)\right\}$, which is also pseudoorthonormal. Then, $e_{1}(x), \ldots, e_{k-1}(x), L(x)$ form a basis for $T S_{x}$. Since $S_{x}$ is totally geodesic, $\alpha\left(e_{t}(x), e_{j}(x)\right)=\alpha\left(e_{i}(x), L\right)=0$, where $\alpha$ is the second fundamental form. Set

$$
A_{\xi}\left(e_{i}\right)=\sum_{j} a_{j i} e_{j}+b_{l} L+\text { a linear combination of } \hat{e}_{k} \text { and } \hat{L}
$$

and

$$
A_{\xi}(L)=\sum_{j} c_{j} e_{j}+b L+\text { a linear combination of } \hat{e}_{k} \text { and } \hat{L} \text {. }
$$

Similarly, $A_{\xi}\left(\hat{e}_{k}\right)$ and $A_{\xi}(L)$ can be given as a linear combination of the basis elements $e_{1}, \ldots, e_{k-1}, L, \hat{L}, \hat{e}_{1}, \ldots, \hat{e}_{n-k-1}$. With respect to this basis, $A_{\xi}$ is represented by an $n \times n$-matrix, which we also denote by the same symbol. In fact, we have

$$
A_{\xi}\left(e_{i}\right)=-\left\langle A_{\xi}\left(e_{i}\right), \hat{L}\right\rangle L+\text { a linear combination of } \hat{e}_{k} \text { 's }
$$

and

$$
A_{\xi}(L)=-\left\langle A_{\xi}(L), \hat{L}\right\rangle L+\text { a linear combination of } \hat{e}_{k} \text { 's. }
$$


Thus,

$$
A_{\xi}=\quad k \quad\left[\begin{array}{cccccc}
0 & \ldots & 0 & * & \cdots & * \\
\vdots & & \vdots & \vdots & & \vdots \\
0 & \ldots & 0 & & & \\
* & \ldots & * & * & \ldots & * \\
0 & \ldots & 0 & * & \ldots & * \\
* & \ldots & * & & & \\
\vdots & & \vdots & \vdots & & \vdots \\
* & \ldots & * & * & \ldots & *
\end{array}\right]
$$

Thus, by interchanging the $k$ th and $(k+1)$ st rows, $A_{\xi}$ has the form:

$$
A_{\xi}=\quad k \quad\left[\begin{array}{cc}
k & n-k \\
O & B \\
C & D .
\end{array}\right]
$$

Here, $O$ is the $k \times k$-zero matrix and $B, C$ and $D$ are $k \times(n-k)$, $(n-k) \times k$ and $(n-k) \times(n-k)$-matrix, respectively. Let $R B$ be the row-reduced echelon matrix of $B$. Then, at least $k-(n-k)=2 k-n$ rows from the bottom of $R B$ must be the zero rows. Similarly, the column-reduced echelon matrix $C C$ of $C$ must have at least $2 k-n$ zero columns on the right side $C$. Denote by $r(x)$ the smaller between the number of the zero rows of $R B$ and the number of the zero columns of $C C$. Applying an appropriate sequence of row operations and column operations, we finally get an $n \times n$-matrix of the following form:

$$
\left[\begin{array}{lc}
O_{r \times r} & O_{r \times(n-r)} \\
O_{(n-r) \times r} & *
\end{array}\right] .
$$

Here $O_{p \times q}$ is the $p \times q$-zero matrix.

Since $\nu(x)$ is the multiplicity of zero as an eigenvalue of $A_{\xi}$ and since the multiplicity of zero is invariant under row and column operations, $A_{\xi}$ must have at least $r(x)$ as its nullity, i.e., $\nu(x) \geq r(x) \geq 2 k-n$.

Theorem 5 is then obtained immediately from Theorem 3 . This result may be regarded as an oversimplified version of "the axiom of sphere" for hypersurfaces. 


\section{REFERENCES}

[1] K. Abe, Application of a Ricatti type differential equation to Riemannian manifolds with totally geodesic distributions, Tohoku Math. J., 25 (1973), 425-444.

[2] __ Some remarks on a class of submanifolds in space forms of non-negative curvature, Math. Ann., 247 (1980), 275-280.

[3] A characterization of totally geodesic hypersurfaces of $S^{n+1}$ and $C P^{n+1}$, Proc. Amer. Math. Soc., 81 No. 4 (1981), 603-606.

[4] S. S. Chern and N. H. Kuiper, Some theorems on the isometric imbedding of compact Riemannian manifolds in Euclidean space, Ann. of Math., 56 (1952), 422-430.

[5] M. Dajczer and P. Dombrowski, Examples of 1-codimensional non-totally geodesic isometric immersions of pseudo-Riemannian space forms with the same positive constant curvature and the same spacelike rank, (to appear).

[6] M. Dajczer and K. Nomizu, On the boundedness of Ricci curvature of an indefinite metric, Bol. Soc. Bras. Mat., 11, \#1 (1980), 25-30.

[7] D. Ferus, Totally geodesic foliations, Math. Ann., 188 (1970), 313-316.

[8] , Isometric immersions of constant curvature manifolds, Math. Ann., 217 (1975), 155-156.

[9] L. Graves, Codimension one isometric immersions between Lorentzian spaces, Trans. Amer. Math. Soc., 252 (1979), 367-392.

[10] _ On codimension one isometric immersions between indefinite space forms, Tsukuba J. Math., 3, No. 2 (1979), 17-29.

[11] L. Graves and K. Nomizu, Isometric immersions of Lorentzian space forms, Math. Ann., 233 (1978), 123-136.

[12] M. Magid, Isometric immersions of Lorentz space with parallel second fundamental forms, Tsukuba J. Math., 8, No. 1 (1984), 31-54.

[13] Shape operators of Einstein hypersurfaces in indefinite space forms, Proc. Amer. Math. Soc., 84 No. 2 (1982), 237-242.

[14] _ Lorentzian isoparametric hypersurfaces, Pacific J. Math., 118 (1985), 165-197.

[15] A. Z. Petrov, Einstein space, Pergamon Press, Hungary, Oxford, and New York (1969).

Received April 2, 1985.

UNIVERSITY OF CONNECTICUT

STORRS, CT 06268

AND

Wellesley College

WELLESLEY, MA 02181 


\section{PACIFIC JOURNAL OF MATHEMATICS EDITORS}

\author{
V. S. VARADARAJAN \\ (Managing Editor) \\ University of California \\ Los Angeles, CA 90024 \\ HeRbert Clemens \\ University of Utah \\ Salt Lake City, UT 84112 \\ R. FINN \\ Stanford University \\ Stanford, CA 94305
}

HERMANN FLASCHKA

University of Arizona

Tucson, AZ 85721

RAMESH A. GANGOLLI

University of Washington

Seattle, WA 98195

VAUGHAN F. R. JONES

University of California

Berkeley, CA 94720

ROBION KIRBY

University of California

Berkeley, CA 94720
C. C. MOORE

University of California

Berkeley, CA 94720

H. SAMELSON

Stanford University

Stanford, CA 94305

HAROLD STARK

University of California, San Diego

La Jolla, CA 92093

\section{ASSOCIATE EDITORS}
R. Arens
E. F. BECKENBACH
B. H. NEUMANN
F. WOLF
K. YoshIDA (1906-1982)

\section{SUPPORTING INSTITUTIONS}

UNIVERSITY OF ARIZONA

UNIVERSITY OF BRITISH COLUMBIA

CALIFORNIA INSTITUTE OF TECHNOLOGY

UNIVERSITY OF CALIFORNIA

MONTANA STATE UNIVERSITY

UNIVERSITY OF NEVADA, RENO

NEW MEXICO STATE UNIVERSITY

OREGON STATE UNIVERSITY
UNIVERSITY OF OREGON

UNIVERSITY OF SOUTHERN CALIFORNIA

TANFORD UNIVERSITY

UNIVERSITY OF HAWAII

UNIVERSITY OF TOKYO

UNIVERSITY OF UTAH

WASHINGTON STATE UNIVERSITY

UNIVERSITY OF WASHINGTON 


\section{Pacific Journal of Mathematics}

Vol. 124, No. $1 \quad$ May, 1986

Kinetsu Abe and Martin Andrew Magid, Relative nullity foliations and indefinite isometric immersions

Erik P. van den Ban, A convexity theorem for semisimple symmetric

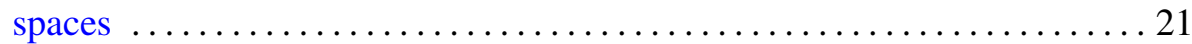

Bo Berndtsson and Thomas Joseph Ransford, Analytic multifunctions, the $\bar{\partial}$-equation, and a proof of the corona theorem .................. 57

Brian Boe and David H. Collingwood, Intertwining operators between

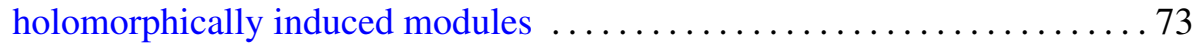

Giuseppe Ceresa and Alessandro Verra, The Abel-Jacobi isomorphism for

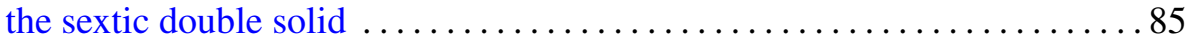

Kun Soo Chang, Jae Moon Ahn and Joo Sup Chang, An evaluation of the conditional Yeh-Wiener integral ........................... 107

Charles Dale Frohman, Minimal surfaces and Heegaard splittings of the

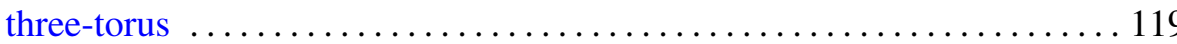

Robert M. Guralnick, Power cancellation of modules $\ldots \ldots \ldots \ldots \ldots \ldots 131$

Kenneth Hardy and Kenneth S. Williams, On the solvability of the Diophantine equation $d V^{2}-2 e V W-d W^{2}=1 \ldots \ldots \ldots \ldots \ldots \ldots \ldots \ldots \ldots$

Ray Alden Kunze and Stephen Scheinberg, Alternative algebras having

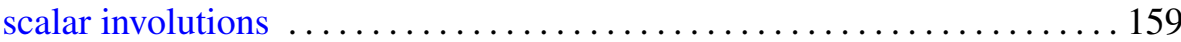

W. B. Raymond Lickorish and Kenneth Millett, The reversing result for the Jones polynomial $\ldots \ldots \ldots \ldots \ldots \ldots \ldots \ldots \ldots$

Guido Lupacciolu, A theorem on holomorphic extension of CR-functions

William Schumacher Massey and Lorenzo Traldi, On a conjecture of K. Murasugi

Dinakar Ramakrishnan, Spectral decomposition of $L^{2}(N \backslash \mathrm{GL}(2), \eta)$

Steven L. Sperber, On solutions of differential equations which satisfy certain algebraic relations 